$1-6-2022$

\title{
Parameter Estimation Based on Double Ranked Set Samples with Applications to Weibull Distribution
}

\author{
Mohamed Abd Elhamed Sabry \\ Cairo University, Cairo, Egypt, mohusss@cu.edu.eg \\ Hiba Zeyada Muhammed \\ Cairo University, Cairo,Egypt, hiba_stat@yahoo.com \\ Mostafa Shaaban \\ The High Institute for Tourism, Hotels \& Computer, Alexandria, Egypt, moushaaban@gmail.com \\ Abd El Hady Nabih \\ Cairo University, Cairo, Egypt, hadi@cu.edu.eg
}

Follow this and additional works at: https://digitalcommons.wayne.edu/jmasm

Part of the Applied Statistics Commons, Social and Behavioral Sciences Commons, and the Statistical Theory Commons

\section{Recommended Citation}

Sabry, M. A. E., Muhammed, H. Z., Shaaban, M., \& Nabih, A. E. H. (2020). Parameter estimation based on double ranked set samples with applications to Weibull distribution. Journal of Modern Applied Statistical Methods, 19(1), eP3070. doi: 10.22237/jmasm/1619482260

This Emerging Scholar is brought to you for free and open access by the Open Access Journals at DigitalCommons@WayneState. It has been accepted for inclusion in Journal of Modern Applied Statistical Methods by an authorized editor of DigitalCommons@WayneState. 


\section{EMERGING SCHOLAR}

\section{Parameter Estimation Based on Double Ranked Set Samples with Applications to Weibull Distribution}

\author{
Mohamed Abd Elhamed Sabry \\ Cairo University \\ Cairo, Egypt
}

\section{Mostafa Shaaban}

The High Institute for Tourism, Hotels \& Computer

Alexandria, Egypt

\author{
Hiba Zeyada Muhammed \\ Cairo University \\ Cairo, Egypt
}

\author{
Abd El Hady Nabih \\ Cairo University \\ Cairo, Egypt
}

In this paper, the likelihood function for parameter estimation based on double ranked set sampling (DRSS) schemes is introduced. The proposed likelihood function is used for the estimation of the Weibull distribution parameters. The maximum likelihood estimators (MLEs) are investigated and compared to the corresponding ones based on simple random sampling (SRS) and ranked set sampling (RSS) schemes. A Monte Carlo simulation is conducted and the absolute relative biases, mean square errors, and efficiencies are compared for the different schemes. It is found that, the MLEs based on DRSS is more efficient than MLE using SRS and RSS for estimating the two parameters of the Weibull distribution (WD).

Keywords: Simple random sampling, ranked set sampling, double ranked set sampling, estimation parameter, maximum likelihood estimation

\section{Introduction}

In 1952, McIntyre (1952) had developed the technique of RSS to find a more efficient method to estimate the mean pasture yields. McIntyre found that the estimator based on ranked set sampling (RSS) is more efficient than SRS. RSS assumed that there will be no errors in ranking the units with respect to the variable of interest which for most practical applications there will imperfect ranking and there will be a loss in efficiency of the estimators based on RSS (see Al-Omari \&

doi: 10.22237/jmasm/1619482260 | Accepted: March 11, 2019; Published: January 6, 2022.

Correspondence: Mostafa Shaaban, moushaaban@gmail.com 


\section{SABRY ET AL}

Jaber, 2010). To reduce the errors in ranking, several modifications on the RSS procedure are made. For example, the extreme ranked set sampling (ERSS) introduced by Samawi et al. (1996), the median ranked set sampling (MRSS) introduced by Muttlak (1997), the moving extreme ranked set sampling (MERSS) introduced by Al-Odat and Al-Saleh (2001), and the multistage ranked set sampling (MSRSS) introduced by Al-Saleh and Al-Omari (2002).

According to Wolfe (2004), the procedure for obtaining the RSS can be summarized as follows:

Step 1: Randomly select $m^{2}$ units from a target population with cumulative distribution function (cdf) and probability density function (pdf) $\mathrm{F}(x ; \theta)$.and $\mathrm{f}(x ; \theta)$, respectively.

Step 2: Allocate the $m^{2}$ selected units as randomly as possible into $m$ sets, each of size $m$.

Step 3: Without yet knowing any values for the variable of interest, rank the units within each set with respect to variable of interest. This may be based on personal professional judgment or done with concomitant variable correlated with the variable of interest.

Step 4: Choose a sample for actual quantification by including the smallest ranked unit in the first set, the second smallest ranked unit in the second set, the process is continued in this way until the largest ranked unit is selected from the last set.

Step 5: Repeat steps 1 through 4 for $r$ cycles to obtain a sample of size $m r$.

RSS uses only one observation, namely $X_{(11) k}$, the lowest observation in the $k^{\text {th }}$ cycle, from this set, then $X_{(22) k}$, the second lowest from another independent set of $m$ observations, and finally $X_{(m m) k}$, the largest observation from a last set of $m$ observations. This process can be described in Figure 1.

\begin{tabular}{ccccc|c}
\hline & & & & & RSS \\
\hline$X_{(11) k}$ & $X_{(12) k}$ & $\cdots$ & $X_{(1(m-1)) k}$ & $X_{(1 m) k}$ & $X_{(11) k}$ \\
$X_{(21) k}$ & $X_{(22) k}$ & $\cdots$ & $X_{(2(m-1)) k}$ & $X_{(2 m) k}$ & $X_{(22) k}$ \\
$\vdots$ & $\vdots$ & & $\vdots$ & $\vdots$ & $\vdots$ \\
$X_{(m 1) k}$ & $X_{(m 2) k}$ & $\cdots$ & $X_{(m(m-1)) k}$ & $X_{(m m) k}$ & $X_{(m m) k}$ \\
\hline
\end{tabular}

Figure 1. Display of $m^{2}$ observations in in the $k^{\text {th }}$ set cycle sets of size $m$ 


\section{PARAMETER ESTIMATION BASED ON DRSS WITH APPLICATIONS}

Let $\left\{X_{(i i) j}, i=1,2, \ldots, m ; j=1,2, \ldots, r\right\}$ be RSS, where $m$ is the set size and $r$

is the number of cycles. In the rest of this paper and for simplification proposes we will use $X_{(i) j}$ instead of $X_{(i i j)}$. Then cdf and pdf of $X_{i, j}$ are given by

$$
\mathrm{F}_{i: m}\left(x_{(i) j} ; \theta\right)=\sum_{t=i}^{m}\left(\begin{array}{c}
m \\
t
\end{array}\right)\left[\mathrm{F}\left(x_{(i) j} ; \theta\right)\right]^{t}\left[1-\mathrm{F}\left(x_{(i) j} ; \theta\right)\right]^{m-t}
$$

and

$$
\mathrm{f}_{i: m}\left(x_{(i) j} ; \theta\right)=\frac{m !}{(i-1) !(m-i) !} \mathrm{f}\left(x_{(i) j} ; \theta\right)\left[\mathrm{F}\left(x_{i ; j} ; \theta\right)^{i-1}\right]\left[1-\mathrm{F}\left(x_{(i) j} ; \theta\right)\right]^{m-i}
$$

respectively, where $-\infty<x_{(i) j}<\infty$. The joint pdf of $x_{(i) j}, i=1,2, \ldots, m, j=1,2, \ldots, r$ is then given by

$$
\mathrm{L}\left(\theta ; X_{\mathrm{R}}\right)=\prod_{i=1}^{m} \mathrm{f}_{i: m}\left(x_{(i) j} ; \theta\right) .
$$

In this paper, we introduce the likelihood function of the double ranked set sampling (DRSS) for estimation of the parameters of different lifetime distributions for the first time. The next section discusses the DRSS procedure. This is followed by an estimation process for the Weibull distribution parameters for different schemes. A simulation study for comparing the DRSS estimators with both SRS and RSS estimators is then presented. Results, conclusions, and final remarks are then given.

\section{Double-Ranked Set Sampling (DRSS)}

According to Al-Saleh and Al-Kadiri (2000), the DRSS is described as follows:

Step 1: Identify $m^{3}$ elements from a target population with cdf and pdf $\mathrm{F}(x ; \theta)$ and $\mathrm{f}(x ; \theta)$, respectively.

Step 2: Divide the $m^{3}$ elements randomly into $m$ sets each of size $m^{2}$ elements and use the usual RSS procedure on each set to obtain $m$ RSS each of size $m$ of the form 


\section{SABRY ET AL}

$$
X_{\mathrm{R}}=\left\{\left\{X_{(1) j}, X_{(2) j}, \ldots, X_{(m) j}\right\}, j=1,2, \ldots, m\right\} .
$$

Step 3: Case I

For even set sizes $(m=2 r)$, select from the first $r$ sets the minimum ranked measurement and from the last $r$ sets select the maximum ranked measurement. The DRSS will be of the form

$$
X_{\mathrm{D}(e)}=\left\{X_{1,1}, X_{1,2}, \ldots, X_{1, r}, X_{m,(r+1)}, X_{m,(r+2)}, \ldots, X_{m, m}\right\},
$$

where

$$
\begin{aligned}
X_{1, j} & =\min \left\{\left\{X_{(1) j}, X_{(2) j}, \ldots, X_{(m) j}\right\}, j=1,2, \ldots, r\right\} \\
X_{m, k} & =\max \left\{\left\{X_{(1) k}, X_{(2) k}, \ldots, X_{(m) k}\right\}, k=r+1, r+2, \ldots, m\right\}
\end{aligned}
$$

\section{Case II}

For odd set sizes $(m=2 r+1)$, select from the first $\mathrm{r}$ sets the minimum ranked measurement, from the $(r+1)^{\text {th }}$ set, select the median and from the last $r$ sets select the maximum measurement. The DRSS will be of the form

$$
X_{\mathrm{D}(o)}=\left\{X_{1,1}, X_{1,2}, \ldots, X_{1, r}, X_{(r+1),(r+1)}, X_{m,(r+2)}, \ldots, X_{m, m}\right\}
$$

where

$$
X_{(r+1),(r+1)}=\operatorname{median}\left\{X_{(1)(r+1)}, X_{(2)(r+1)}, \ldots, X_{(m)(r+1)}\right\} .
$$

\section{Joint Probability Distribution of DRSS}

In this section we will derive the joint probability distribution of a modified DRSS. It can be easily shown that for the minimum, maximum, and median order statistics, the cdfs are given by

$$
\mathrm{F}_{1: n}(x ; \theta)=\mathrm{P}_{1: n}(X \leq x)=1-[1-\mathrm{F}(x ; \theta)]^{n}
$$




\section{PARAMETER ESTIMATION BASED ON DRSS WITH APPLICATIONS}

$$
\begin{aligned}
\mathrm{F}_{n: n}(X ; \theta) & =[\mathrm{F}(x ; \theta)]^{n} \\
\mathrm{~F}_{\text {med: } n}(x ; \theta) & =\frac{(2 r+1) !}{(r) !(r) !} \sum_{w=0}^{r} \frac{(-1)^{w}\left(\begin{array}{c}
r \\
w
\end{array}\right)}{r+w+1}\left(\mathrm{~F}\left(x_{w} ; \theta\right)\right)^{r+w+1}
\end{aligned}
$$

for odd sample size $n=2 r+1$.

For a DRSS with even set sizes $m=2 r$ defined in equation (3), the cdf and the pdf of $X_{1: m}^{j}, j=1,2, \ldots, r$ are given by

$$
\begin{aligned}
& \mathrm{F}_{X_{1, j}}\left(x_{1, j} ; \theta\right)=\mathrm{P}_{X_{1, j}}\left(X_{1, j} \leq x_{1, j}\right)=1-\left[1-\mathrm{F}_{1: m}\left(x_{1, j} ; \theta\right)\right]^{m} \\
& \mathrm{f}_{X_{1, j}}\left(x_{1, j} ; \theta\right)=m \mathrm{f}_{1: m} \mathrm{P}_{X_{1, j}}\left(x_{1, j} ; \theta\right)\left[1-\mathrm{F}_{1: m}\left(x_{1, j} ; \theta\right)\right]^{m-1}
\end{aligned}
$$

respectively, and the likelihood function of the subsample $X_{1,1}, X_{1,2}, \ldots, X_{(1, r)}$ will be given by

$$
\mathrm{L}_{1: m}(\theta)=\prod_{j=1}^{r}\left(m \mathrm{f}_{1: m}\left(x_{1, j} ; \theta\right)\left[1-\mathrm{F}_{1: m}\left(x_{1, j} ; \theta\right)\right]^{m-1}\right) .
$$

Similarly, the cdf and pdf of $X_{m: m}^{k}$ are given by

$$
\begin{aligned}
& \mathrm{F}_{X_{m, k}}\left(x_{m, k} ; \theta\right)=\mathrm{P}_{X_{m, k}}\left(X_{m, k} \leq x_{m, k}\right)=\left[\mathrm{F}_{m: m}\left(x_{m, k} ; \theta\right)\right]^{m} \\
& \mathrm{f}_{X_{m, k}}\left(x_{m, k} ; \theta\right)=m \mathrm{f}_{m: m}\left(x_{m, k} ; \theta\right)\left[\mathrm{F}_{m: m}\left(x_{m, k} ; \theta\right)\right]^{m-1}
\end{aligned}
$$

respectively, and the joint pdf of the random vector $X_{m: m}^{r+1}, X_{m: m}^{r+2}, \ldots, X_{m: m}^{m}$ is defined as

$$
\mathrm{L}_{m: m}(\theta)=\prod_{k=r+1}^{m}\left(m \mathrm{f}_{m: m}\left(x_{m, k} ; \theta\right)\left[\mathrm{F}_{m: m}\left(x_{m, k} ; \theta\right)\right]^{m-1}\right) .
$$

From equations (5) and (6), the likelihood function for an even size DRSS will be of the form 


\section{SABRY ET AL}

$$
\begin{aligned}
\mathrm{L}\left(\theta ; X_{\mathrm{D}(e)}\right)= & {\left[\prod_{j=1}^{r} \mathrm{f}_{X_{1, j}}\left(x_{1, j} ; \theta\right)\right] \times\left[\prod_{k=r+1}^{m} \mathrm{f}_{X_{m, k}}\left(x_{m, k} ; \theta\right)\right] } \\
= & {\left[\prod_{j=1}^{r}\left(m \mathrm{f}_{1: m}\left(x_{1, j} ; \theta\right)\left[1-\mathrm{F}_{1: m}\left(x_{1, j} ; \theta\right)\right]^{m-1}\right)\right] } \\
& \times\left[\prod_{k=r+1}^{m}\left(m \mathrm{f}_{m: m}\left(x_{m, k} ; \theta\right)\left[\mathrm{F}_{m: m}\left(x_{m, k} ; \theta\right)\right]^{m-1}\right)\right]
\end{aligned}
$$

When $m$ is odd, say $m=2 r+1$, the cdf and pdf of $X_{(r+1),(r+1)}$ is given by

$$
\mathrm{F}_{X_{(r+1),(r+1)}}\left(x_{(r+1),(r+1)} ; \theta\right)=\frac{(2 r+1) !}{(r) !(r) !} \sum_{l=0}^{r} \frac{(-1)^{l}\left(\begin{array}{l}
r \\
l
\end{array}\right)}{r+l+1}\left(\mathrm{~F}_{r+1: m}\left(x_{(r+1),(r+1)} ; \theta\right)\right)^{r+l+1}
$$

and

$$
\begin{aligned}
\mathrm{f}_{X_{(r+1),(r+1)}}\left(x_{(r+1),(r+1)} ; \theta\right)= & \frac{(2 r+1) !}{(r) !(r) !} \mathrm{f}_{r+1: m}\left(x_{(r+1),(r+1)} ; \theta\right) \\
& \times\left(\mathrm{F}_{r+1: m}\left(x_{(r+1),(r+1)} ; \theta\right)\left(1-\mathrm{F}_{r+1: m}\left(x_{(r+1),(r+1)} ; \theta\right)\right)\right)^{r}
\end{aligned}
$$

From equations (5), (6), and (9), the likelihood function for an odd size DRSS will be of the form

$$
\begin{aligned}
\mathrm{L}_{X_{\mathrm{D}(o)}}(\theta)= & {\left[\prod_{j=1}^{r} \mathrm{f}_{X_{1, j}}\left(x_{1, j} ; \theta\right)\right]\left[\prod_{k=r+1}^{m} \mathrm{f}_{X_{m, k}}\left(x_{m, k} ; \theta\right)\right]\left(\mathrm{f}_{X_{(r+1),(r+1)}}\left(X_{(r+1),(r+1)} ; \theta\right)\right) } \\
= & {\left[\prod_{j=1}^{r} m \mathrm{f}_{1: m}\left(x_{1, j} ; \theta\right)\left[1-\mathrm{F}_{1: m}\left(x_{1, j} ; \theta\right)\right]^{m-1}\right] } \\
& \times\left[\prod_{k=r+1}^{m} m \mathrm{f}_{m: m}\left(x_{m, k} ; \theta\right)\left[\mathrm{F}_{m: m}\left(x_{m, k} ; \theta\right)\right]^{m-1}\right] \\
& \times \frac{(2 r+1) !}{(r) !(r) !} \mathrm{f}_{r+1: m}\left(x_{(r+1),(r+1)} ; \theta\right) \\
& \times\left(\mathrm{F}_{r+1: m}\left(x_{(r+1),(r+1)} ; \theta\right)\left(1-\mathrm{F}_{r+1: m}\left(x_{(r+1),(r+1)} ; \theta\right)\right)\right)^{r}
\end{aligned}
$$




\section{PARAMETER ESTIMATION BASED ON DRSS WITH APPLICATIONS}

For more information about distributions of order statistics, the reader is recommended to David and Nagaraja (2003) and Barry et al. (2008).

\section{Special Cases}

In this section two special cases regarding the DRSS and the likelihood function are considered. These cases can provide more flexible schemes based on DRSS samples.

\section{Case I}

We can choose $X_{\mathrm{D}}=\left\{X_{1,1}, X_{1,2}, \ldots, X_{1, m}\right\}$ which will lead to a right DRSS with likelihood function

$$
\mathrm{L}_{X_{\mathrm{DR}}}(\theta)=\prod_{j=1}^{m} m \mathrm{f}_{1: m}\left(x_{1, j} ; \theta\right)\left[1-\mathrm{F}_{1: m}\left(x_{1, j} ; \theta\right)\right]^{m-1}
$$

\section{Case II}

We can choose $X_{\mathrm{DR}}=\left\{X_{m, 1}, X_{m, 2}, \ldots, X_{m, m}\right\}$ which will lead to a left DRSS with likelihood function

$$
\mathrm{L}_{X_{\mathrm{DL}}}(\theta)=\prod_{j=1}^{m} m \mathrm{f}_{m: m}\left(x_{m, j} ; \theta\right)\left[\mathrm{F}_{m: m}\left(x_{m, j} ; \theta\right)\right]^{m-1} .
$$

\section{Estimation of the Weibull Distribution Parameters}

The Weibull distribution introduced by the Swedish physicist Weibull (1951), is one of the most widely used lifetime distributions in reliability engineering. It is a versatile distribution that can take on the characteristics of other types of distributions, where $\beta$ is a shape parameter and $\lambda$ is a scale parameter based on the value of the shape parameter. The cdf, pdf, and the quantile functions of the Weibull distribution are given by

$$
\begin{gathered}
\mathrm{F}(x ; \lambda, \beta)=1-e^{-\lambda x^{\beta}}, \\
\mathrm{f}(x ; \lambda, \beta)=\lambda \beta x^{\beta-1} e^{-\lambda x^{\beta}},
\end{gathered}
$$




\section{SABRY ET AL}

and

$$
\mathrm{Q}(u)=\left[\frac{-\ln (1-u)}{\lambda}\right]^{\frac{1}{\beta}},
$$

respectively, where $x>0, \lambda>0, \beta>0$, and $0<u<1$.

\section{Estimation Based on SRS}

Let $X_{1}, X_{2}, \ldots, X_{n}$ be independent and identically distributed random variables from Weibull distribution with pdf given in equation (10). The likelihood function of $\lambda$ and $\beta$ is given by

$$
\mathrm{L}(\lambda, \beta ; x)=\prod_{i=1}^{n} \lambda \beta x_{i}^{\beta-1} e^{-\lambda x_{i}^{\beta}}
$$

and the log likelihood function is then derived as

$$
\ell(\lambda, \beta)=n \log \lambda+n \log \beta+(\beta-1) \sum_{i=1}^{n} \log x_{i}-\sum_{i=1}^{n} \lambda x_{i}^{\beta}
$$

Now, the likelihood equations are

$$
\frac{n}{\hat{\lambda}}-\sum_{i=1}^{n} x_{i}^{\hat{\beta}}=0
$$

and

$$
\frac{n}{\hat{\beta}}+\sum_{i=1}^{n} \log x_{i}-\sum_{i=1}^{n} \hat{\lambda} x_{i}^{\hat{\beta}} \log x_{i}=0
$$

Equation (14) don't have a closed form solution. Therefore, numerical techniques are used to solve for the MLEs of the scale and shape parameters. 


\section{PARAMETER ESTIMATION BASED ON DRSS WITH APPLICATIONS}

\section{Estimation Based on RSS}

Let $\left\{X_{i}^{j}, i=1,2, \ldots, n ; j=1,2, \ldots, r\right\}$ be a ranked set sample with cdf and pdf given in equations (1) and (2), where $\mathrm{n}$ is the set size, $r$ is the number of cycles, and $m=n r$. The Likelihood function of the RSS sample for Weibull data is given by

$$
\begin{aligned}
\mathrm{L}_{r}(\lambda, \beta ; x) \\
=\prod_{j=1}^{r} \prod_{i=1}^{n} C_{i} \mathrm{f}\left(x_{(i) j} ; \lambda, \beta\right)\left[\mathrm{F}\left(x_{(i) j} ; \lambda, \beta\right)^{i-1}\right]\left[1-\mathrm{F}\left(x_{(i) j} ; \lambda, \beta\right)\right]^{n-i} \\
=\prod_{j=1}^{r} \prod_{i=1}^{n} C_{i}\left(\lambda \beta\left(x_{(i) j}\right)^{\beta-1} e^{-\lambda\left(x_{(i) j}\right)^{\beta}}\right)\left(1-e^{-\lambda\left(x_{(i) j}\right)^{\beta}}\right)^{i-1}\left(e^{-\lambda\left(x_{(i) j}\right)^{\beta}}\right)^{n-i}
\end{aligned}
$$

where

$$
C_{i}=\frac{n !}{(i-1) !(n-i) !}
$$

The log likelihood function can be derived directly as follows:

$$
\begin{aligned}
\ell_{r}(\lambda, \beta) \propto n r \log \lambda & +n r \log \beta+(\beta-1) \sum_{j=1}^{r} \sum_{i=1}^{n} \log x_{(i) j} \\
& -\sum_{j=1}^{r} \sum_{i=1}^{n}(n-i+1) \lambda\left(x_{(i) j}\right)^{\beta}+\sum_{j=1}^{r} \sum_{i=1}^{n}(i-1) \log \left(1-e^{-\lambda\left(x_{(i) j}\right)^{\beta}}\right)
\end{aligned}
$$

The likelihood equations become

$$
\frac{n r}{\hat{\lambda}}-(n-i+1) \sum_{j=1}^{r} \sum_{i=1}^{n}\left(x_{(i) j}\right)^{\hat{\beta}}+(i-1) \sum_{j=1}^{r} \sum_{i=1}^{n} \frac{\left(x_{(i) j}\right)^{\hat{\beta}} e^{-\lambda\left(x_{(i) j}\right)^{\hat{\beta}}}}{1-e^{-\hat{\lambda}\left(x_{(i) j}\right)^{\hat{\beta}}}}=0
$$

and 


\section{SABRY ET AL}

$$
\begin{aligned}
\frac{n r}{\hat{\beta}}+\sum_{j=1}^{r} \sum_{i=1}^{n} \log x_{(i) j} & -(n-i+1) \sum_{j=1}^{r} \sum_{i=1}^{n} \hat{\lambda}\left(x_{(i) j}\right)^{\hat{\beta}} \log x_{(i) j} \\
& +(i-1) \sum_{j=1}^{r} \sum_{i=1}^{n} \frac{\lambda\left(x_{(i) j}\right)^{\hat{\beta}} e^{-\hat{\lambda}\left(x_{(i) j}\right)^{\hat{\beta}}} \log x_{(i) j}}{1-e^{-\hat{\lambda}\left(x_{(i) j}\right)^{\hat{\beta}}}}=0
\end{aligned}
$$

These two nonlinear equations can't be solved analytically and will be solved numerically.

\section{Estimation Based on DRSS}

According to equations (7) and (10), the likelihood function for the Weibull distribution is derived as follows:

Case I. $\quad m$ even $(m=2 r)$

$$
\mathrm{L}_{\mathrm{D}(e)}(\theta)=\left[\prod_{j=1}^{r} m \mathrm{f}_{1: m}\left(x_{1, j}\right)\left[1-\mathrm{F}_{1: m}\left(x_{1, j}\right)\right]^{m-1}\right]\left[\prod_{k=r+1}^{m} m \mathrm{f}_{m: m}\left(x_{m, k}\right)\left[\mathrm{F}_{m: m}\left(x_{m, k}\right)\right]^{m-1}\right] .
$$

where $\quad \mathrm{f}_{1: m}\left(x_{j}\right)=m \mathrm{f}\left(x_{1, j}\right)\left[1-\mathrm{F}\left(x_{1, j}\right)\right]^{\mathrm{m}-1}, \quad \mathrm{~F}_{1: m}\left(x_{1, j}\right)=1-\left[1-\mathrm{F}\left(x_{1, j}\right)\right]^{m}$, $\mathrm{f}_{m: m}\left(x_{k}\right)=m \mathrm{f}\left(x_{m, k}\right)\left[\mathrm{F}\left(x_{m, k}\right)\right]^{m-1}$ and $\mathrm{F}_{m: m}\left(x_{m, k}\right)=\left[\mathrm{F}\left(x_{m, k}\right)\right]^{m}$. The likelihood function is then given by 
PARAMETER ESTIMATION BASED ON DRSS WITH APPLICATIONS

$$
\begin{aligned}
& \mathrm{L}_{\mathrm{D}(e)}(\theta)=\left[\prod_{j=1}^{r} m\left(m \mathrm{f}\left(x_{1, j}\right)\left[1-F\left(x_{1, j}\right)\right]^{m-1}\right)\left[\left[1-\mathrm{F}\left(x_{1, j}\right)\right]^{m}\right]^{m-1}\right] \\
& \times\left[\prod_{k=r+1}^{m} m\left(m \mathrm{f}\left(x_{m, k}\right)\left[\mathrm{F}\left(x_{m, k}\right)\right]^{m-1}\right)\left[\left[\mathrm{F}\left(x_{m, k}\right)\right]^{m}\right]^{m-1}\right] \\
&= {\left[\prod_{j=1}^{r} m\left(m \lambda \beta x_{1, j}^{\beta-1} e^{-\lambda x_{1, j}^{\beta}}\left(e^{-\lambda x_{1, j}^{\beta}}\right)^{m-1}\right)\left(e^{-\lambda x_{1, j}^{\beta}}\right)^{m(m-1)}\right] } \\
& \times\left[\prod_{k=r+1}^{m} m\left(m \lambda \beta x_{m, k}^{\beta-1}\left(e^{-\lambda x_{m, k}^{\beta}}\right)\right)\left(1-e^{-\lambda x_{m, k}^{\beta}}\right)^{m-1}\left[\left(1-e^{-\lambda x_{m, k}^{\beta}}\right)^{m}\right]^{(m-1)}\right] \\
&= {\left[\prod_{j=1}^{r} m\left(m \lambda \beta x_{1, j}^{\beta-1}\left(e^{-\lambda m^{2} x_{1, j}^{\beta}}\right)\right)\right] } \\
& \times\left[\prod_{k=r+1}^{m} m\left(m \lambda \beta x_{m, k}^{\beta-1}\left(e^{-\lambda x_{m, k}^{\beta}}\right)\right)\left(1-e^{-\lambda x_{m, k}^{\beta}}\right)^{\left(m^{2}-1\right)}\right] \\
&= m^{2 m} \lambda^{m} \beta^{m}\left(\prod_{j=1}^{r} x_{1, j}^{\beta-1}\right)\left(\prod_{k=r+1}^{m} x_{m, k}^{\beta-1}\right)\left(e^{-\lambda m^{2} \sum_{j=1}^{r} x_{1, j}^{\beta}}\right)\left(e^{-\lambda \sum_{k=r+1}^{m} x_{m, k}^{\beta}}\right) \\
& \times\left(\prod_{k=r+1}^{m}\left(1-e^{-\lambda x_{m, k}^{\beta}}\right)^{\left(m^{2}-1\right)}\right]
\end{aligned}
$$

Then, the associated log-likelihood function is obtained as

$$
\begin{aligned}
\ell_{\mathrm{D}(e)}=2 m \log m & +m \log \lambda+m \log \beta+(\beta-1) \sum_{j=1}^{r} \log x_{1, j}+(\beta-1) \sum_{k=r+1}^{m} \log x_{m, k} \\
& -\lambda m^{2} \sum_{j=1}^{r} x_{1, j}^{\beta}-\lambda \sum_{k=r+1}^{m} x_{m, k}^{\beta}+\left(m^{2}-1\right) \sum_{k=r+1}^{m} \log \left(1-e^{-\lambda x_{m, k}^{\beta}}\right)
\end{aligned}
$$

and the likelihood equations are given by

$$
\frac{\partial \ell_{\mathrm{D}(e)}}{\partial \lambda}=\frac{m}{\lambda}-m^{2} \sum_{j=1}^{r} x_{1, j}^{\beta}-\sum_{k=r+1}^{m} x_{m, k}^{\beta}+\left(m^{2}-1\right) \sum_{k=r+1}^{m} \frac{x_{m, k}^{\beta} e^{-\lambda x_{m, k}^{\beta}}}{1-e^{-\lambda x_{m, k}^{\beta}}}
$$

and 


\section{SABRY ET AL}

$$
\begin{aligned}
\frac{\partial \ell_{\mathrm{D}(e)}}{\partial \beta}=\frac{m}{\beta}+\sum_{j=1}^{r} & \log x_{1, j}+\sum_{k=r+1}^{m} \log x_{m, k}-\lambda m^{2} \sum_{j=1}^{r} x_{1, j}^{\beta} \log x_{1, j} \\
& -\lambda \sum_{k=r+1}^{m} x_{m, k}^{\beta} \log x_{m, k}+\left(m^{2}-1\right) \sum_{k=r+1}^{m} \frac{\lambda x_{m, k}^{\beta} \log x_{m, k} e^{-\lambda x_{m, k}^{\beta}}}{1-e^{-\lambda x_{m, k}^{\beta}}}
\end{aligned}
$$

The maximum likelihoods estimators (MLEs) of $\lambda$ and $\beta$, denoted by $\hat{\lambda}$ and $\hat{\beta}$, can be computed by solving the above likelihood equations. The above equations cannot give a closed form solutions and for that, some numerical methods will be employed in order to obtain the desired estimates.

Case II. $\quad m$ odd $(m=2 r+1)$

$$
\begin{aligned}
\mathrm{L}_{\mathrm{D}(o)}(\theta)= & {\left[\prod_{j=1}^{r} m \mathrm{f}_{1: m}\left(x_{1, j}\right)\left[1-\mathrm{F}_{1: m}\left(x_{1, j}\right)\right]^{m-1}\right]\left[\prod_{k=r+2}^{m} m \mathrm{f}_{m: m}\left(x_{m, k}\right)\left[\mathrm{F}_{m: m}\left(x_{m, k}\right)\right]^{m-1}\right] } \\
& \times\left[\frac{(2 r+1) !}{(r) !(r) !} \mathrm{f}_{r+1: m}\left(x_{(r+1),(r+1)}\right)\left(\mathrm{F}_{r+1: m}\left(x_{(r+1),(r+1)}\right)\left(1-\mathrm{F}_{r+1: m}\left(x_{(r+1),(r+1)}\right)\right)\right)^{r}\right] \\
= & {\left[\prod_{j=1}^{r} m \mathrm{f}_{1: m}\left(x_{1, j}\right)\left[1-\mathrm{F}_{1: m}\left(x_{1, j}\right)\right]^{m-1}\right]\left[\prod_{k=r+2}^{m} m \mathrm{f}_{m: m}\left(x_{m, k}\right)\left[\mathrm{F}_{m: m}\left(x_{m, k}\right)\right]^{m-1}\right] } \\
& \times\left[\frac { ( 2 r + 1 ) ! } { ( r ) ! ( r ) ! } \left(\left[\frac{m !}{r ! r !} \mathrm{f}\left(x_{(r+1),(r+1)}\right)\left(\mathrm{F}\left(x_{(r+1),(r+1)}\right)\right)^{r}\left(1-\mathrm{F}\left(x_{(r+1),(r+1)}\right)\right)^{r}\right)\right.\right. \\
& \times\left(\mathrm{F}_{r+1: m}\left(x_{(r+1),(r+1)}\right)\left(1-\mathrm{F}_{r+1: m}\left(x_{(r+1),(r+1)}\right)\right)\right)^{r}
\end{aligned}
$$

Since

$$
\mathrm{F}_{r+1: m}\left(x_{(r+1),(r+1)}\right)=\sum_{t=r+1}^{m}\left(\begin{array}{c}
m \\
t
\end{array}\right)\left(\mathrm{F}\left(x_{(r+1),(r+1)}\right)\right)^{t}\left(1-\mathrm{F}\left(x_{(r+1),(r+1)}\right)\right)^{m-t}
$$

Then 


\section{PARAMETER ESTIMATION BASED ON DRSS WITH APPLICATIONS}

$$
\begin{aligned}
& \mathrm{L}_{\mathrm{D}(o)}(\theta)=\left[\prod_{j=1}^{r} m\left(m \lambda \beta x_{1, j}^{\beta-1}\left(e^{-\lambda m^{2} x_{1, j}^{\beta}}\right)\right)\right] \\
& \times\left[\prod_{k=r+2}^{m} m\left(m \lambda \beta x_{m, k}^{\beta-1}\left(e^{-\lambda x_{m, k}^{\beta}}\right)\right)\left(1-e^{-\lambda x_{m, k}^{\beta}}\right)^{\left(m^{2}-1\right)}\right] \\
& \times\left[\frac{(2 r+1) !}{(r) !(r) !}\left(\frac{m !}{r ! r !}\left(\lambda \beta x_{(r+1),(r+1)}^{\beta-1} e^{-\lambda(r+1) x_{(r+1),(r+1)}^{\beta}}\right)\left(1-e^{-\lambda x_{(r+1),(r+1)}^{\beta}}\right)^{r}\right)\right. \\
& \times\left(\mathrm{F}_{r+1: m}\left(x_{(r+1),(r+1)}\right)\right)^{r}\left(1-\mathrm{F}_{r+1: m}\left(x_{(r+1),(r+1)}\right)\right)^{r} \\
& =m^{2(m-1)} \lambda^{m} \beta^{m}\left(\prod_{j-1}^{r} x_{1, j}^{\beta-1}\right)\left(\prod_{k=r+2}^{m} x_{m, k}^{\beta-1}\right)\left(e^{-\lambda m^{2} \sum_{j=1}^{r} x_{1, j}^{\beta}}\right) \\
& \times\left(e^{-\lambda \sum_{k=r+2}^{m} x_{m, k}^{\beta}}\right) \prod_{k=r+2}^{m}\left(1-e^{-\lambda x_{m, k}^{\beta}}\right)^{\left(m^{2}-1\right)} \\
& \times\left[\frac{(2 r+1) !}{(r) !(r) !}\left(\frac{m !}{r ! r !}\left(x_{(r+1),(r+1)}^{\beta-1} e^{-\lambda(r+1) x_{(r+1),(r+1)}^{\beta}}\right)\left(1-e^{-\lambda x_{(r+1),(r+1)}^{\beta}}\right)^{r}\right)\right. \\
& \times\left(\mathrm{F}_{r+1: m}\left(x_{(r+1),(r+1)}\right)\right)^{r}\left(1-\mathrm{F}_{r+1: m}\left(x_{(r+1),(r+1)}\right)\right)^{r}
\end{aligned}
$$

Then, the associated log-likelihood function is obtained as

$$
\begin{aligned}
\ell_{\mathrm{D}(o)}=2( & m-1) \log m+m \log \lambda+m \log \beta+(\beta-1) \sum_{j=1}^{r} \log x_{1, j} \\
& +(\beta-1) \sum_{k=r+2}^{m} \log x_{m, k}-\lambda m^{2} \sum_{j=1}^{r} x_{1, j}^{\beta}-\lambda \sum_{k=r+2}^{m} x_{m, k}^{\beta} \\
& +\left(m^{2}-1\right) \sum_{k=r+2}^{m} \log \left(1-e^{-\lambda x_{m, k}^{\beta}}\right)+\log \left(\frac{(2 r+1) !}{(r) !(r) !} \frac{m !}{r ! r !}\right) \\
& +(\beta-1) \log x_{(r+1),(r+1)}-\lambda(r+1) x_{(r+1),(r+1)}^{\beta}+r \log \left(1-e^{-\lambda x_{(r+1),(r+1)}^{\beta}}\right) \\
& +r \log \left(\mathrm{F}_{r+1: m}\left(x_{(r+1),(r+1)}\right)\right)+r \log \left(1-\mathrm{F}_{r+1: m}\left(x_{(r+1),(r+1)}\right)\right)
\end{aligned}
$$

and the likelihood equations in this case are given by 


\section{SABRY ET AL}

$$
\begin{aligned}
\frac{\partial \ell_{\mathrm{D}(o)}}{\partial \lambda}=\frac{m}{\lambda} & -m^{2} \sum_{j=1}^{r} x_{1, j}^{\beta}-\sum_{k=r+2}^{m} x_{m, k}^{\beta}+\left(m^{2}-1\right) \sum_{k=r+2}^{m}\left(\frac{x_{m, k}^{\beta} e^{-\lambda x_{m, k}^{\beta}}}{1-e^{-\lambda x_{m, k}^{\beta}}}\right) \\
& -(r+1) x_{(r+1),(r+1)}^{\beta}+r\left(\frac{x_{(r+1),(r+1)}^{\beta} e^{-\lambda x_{(r+1),(r+1)}^{\beta}}}{1-e^{-\lambda x_{(r+1)(r+1)}^{\beta}}}\right) \\
& +\mathrm{F}_{\lambda}\left(\frac{1-2 \mathrm{~F}_{r+1: m}\left(x_{(r+1),(r+1)}\right)}{\mathrm{F}_{r+1: m}\left(x_{(r+1),(r+1)}\right)\left(1-\mathrm{F}_{r+1: m}\left(x_{(r+1),(r+1)}\right)\right)}\right)
\end{aligned}
$$

and

$$
\begin{aligned}
\frac{\partial \ell_{\mathrm{D}(o)}}{\partial \beta}= & \frac{m}{\beta}+\sum_{j=1}^{r} \log x_{1, j}+\sum_{k=r+2}^{m} \log x_{m, k}-\lambda m^{2} \sum_{j=1}^{r} x_{1, j}^{\beta} \log x_{1, j} \\
& -\lambda \sum_{k=r+2}^{m} x_{m, k}^{\beta} \log x_{m, k}+\left(m^{2}-1\right) \sum_{k=r+2}^{m} \frac{\lambda x_{m, k}^{\beta} \log x_{m, k} e^{-\lambda x_{m, k}^{\beta}}}{1-e^{-\lambda x_{m, k}^{\beta}}} \\
& +\log x_{(r+1),(r+1)}-\lambda(r+1)\left(\log x_{(r+1),(r+1)}\right) x_{(r+1),(r+1)}^{\beta} \\
& +r\left(\frac{\lambda x_{(r+1),(r+1)}^{\beta} \log x_{(r+1),(r+1)} e^{-\lambda x_{(r+1),(r+1)}^{\beta}}}{1-e^{-\lambda x_{(r+1)(, r+1)}^{\beta}}}\right) \\
& +\mathrm{F}_{\beta}\left(\frac{1-2 \mathrm{~F}_{r+1: m}\left(x_{(r+1),(r+1)}\right)}{\mathrm{F}_{r+1: m}\left(x_{(r+1),(r+1)}\right)\left(1-\mathrm{F}_{r+1: m}\left(x_{(r+1),(r+1)}\right)\right)}\right)
\end{aligned}
$$

Where

$$
\mathrm{F}_{\lambda}=\frac{\partial \mathrm{F}_{r+1: m}}{\partial \lambda}\left(x_{(r+1),(r+1)}\right) \text { and } \quad \mathrm{F}_{\beta}=\frac{\partial \mathrm{F}_{r+1: m}}{\partial \beta}\left(x_{(r+1),(r+1)}\right) .
$$

\section{Simulation Study}

In this section, we study a Monte Carlo simulation to compare the performance of the Maximum Likelihood method under complete sample, ranked sample and 


\section{PARAMETER ESTIMATION BASED ON DRSS WITH APPLICATIONS}

double ranked sample. The data were generated from the Weibull distribution for different values of $\lambda$ and $\beta$. The simulation algorithm is as follows:

For complete samples

1. Generate $m$ random samples from the Weibull distribution using the quantile function defined in equation (13) with 10000 replicates.

2. For different sample sizes $m=6,9,10,15,20,25$, and 30, and different parameter values for $\lambda$ and $\beta$ are $\lambda=0.5,1.5$, and $3 ; \beta=0.5,1.5$, and 3 . Obtain the MLE.

3. Calculate the bias and mean square errors (MSE) of the estimates derived from equations (14) and (15).

For ranked set samples

1. Generate $n$ random samples from the Weibull distribution using the quantile function defined in equation (13) with 10000 replicates.

2. Use the RSS method to simulate RSS samples as mentioned earlier.

3. Repeat steps 1 and $2 r$ (no. of cycles) times such that $m=n r$.

4. Obtain the MLE by solving equations (17) and (18) simultaneously and calculate the bias, MSE, and relative efficiency of the RSS estimators compared to the SRS estimators where the relative efficiency of $\hat{\theta}_{2}$ compared with $\hat{\theta}_{1}$ is defined as

$$
\operatorname{Eff}\left(\hat{\theta}_{1}, \hat{\theta}_{2}\right)=\frac{\operatorname{MSE}\left(\hat{\theta}_{1}\right)}{\operatorname{MSE}\left(\hat{\theta}_{2}\right)}
$$

For double ranked set samples

1. Generate $m^{3}$ random samples from the Weibull distribution using the quantile function defined in equation (13) with 10000 replicates.

2. Use the DRSS method to simulate DRSS samples as mentioned earlier for even and odd sample sizes and obtain the MLE equations (20) and (21) for odd samples and equation (23) and (24) for even samples.

3. Calculate the bias, MSE, and relative efficiency of the DRSS estimators compared to the RSS estimators. 


\section{SABRY ET AL}

The results of the simulation study are reported in Tables 1-3. From the tables it can be seen that

1. As the sample size increases the MSE decreases for both $\lambda$ and $\beta$ parameters.

2. For fixed $m$ and $\lambda$ as $\beta$ increases the MSE for both $\lambda$ and $\beta$ increases.

3. As $\lambda$ increases the MSE for both $\lambda$ and $\beta$ increases.

4. The relative efficiencies for both DRSS estimators are higher than 1, indicating better estimators compared to the both RSS and SRS estimators.

5. An overfitting problem may be an issue when using the proposed DRSS likelihood functions.

\section{Conclusion}

The likelihood function for DRSS schemes were derived and maximum likelihood estimators for the Weibull distribution were studied based on SRS, RSS and DRSS. It is clear from the simulation results that the likelihood function used to estimate the parameters of the Weibull distribution based on DRSS showed relatively efficient estimates compared to RSS estimators and the authors recommend using the DRSS likelihood function proposed for estimating distribution parameters.

\section{References}

Al-Odat, M. T., \& Al-Saleh, M. F. (2001). A variation of ranked set sampling. Journal of Applied Statistical Science, 10(2), 137-146.

Al-Omari, A. I., \& Jaber, K. (2010). Improvement in estimating the population mean in double extreme ranked set sampling. International Mathematical Forum, 5(26), 1265-1275. Retrieved from http://www.mhikari.com/imf-2010/25-28-2010/alomariIMF25-28-2010.pdf

Al-Saleh, M. F., \& Al-Kadiri, M. A. (2000). Double ranked set sampling. Statistics \& Probability Letters, 48(2), 205-212. doi: 10.1016/S01677152(99)00206-0

Al-Saleh, M. F., \& Al-Omari A. I. (2002). Multistage ranked set sampling. Journal of Statistical Planning and Inference, 102(2), 273-286. doi: 10.1016/S0378-3758(01)00086-6 


\section{PARAMETER ESTIMATION BASED ON DRSS WITH APPLICATIONS}

Barry, C. A., Balakrishnan, N., \& Nagaraja, H. N. (2008). A first course in order statistics. Philadelphia, PA: Society for Industrial and Applied Mathematics. doi: 10.1137/1.9780898719062

David, H. A., \& Nagaraja, H. N. (2003). Order statistics ( $3^{\text {rd }}$ edition). New York: John Wiley \& Sons, Inc. doi: 10.1002/0471722162

McIntyre, G. A. (1952). A method for unbiased selective sampling, using ranked sets. Australian Journal of Agricultural Research, 3(4), 385-390. doi: 10.1071/AR9520385

Muttlak, H. A. (1997). Median ranked set sampling. Journal of Applied Statistics Sciences, 6, 245-255.

Samawi, H. M., Ahmed, M. S., \& Abu-Dayyeh, W. A. (1996). Estimating the population mean using extreme ranked set sampling. Biometrical Journal, 38(5), 577-586. doi: 10.1002/bimj.4710380506

Weibull, W. (1951). A statistical distribution function of wide applicability. Journal of Applied Mechanics, 18(3), 293-297.

Wolfe, D. A. (2004). Ranked set sampling: An approach to more efficient data collection. Statistical Science, 19(4), 636-643. doi:

10.1214/088342304000000369 
SABRY ET AL

\section{Appendix A: Tables}

Table 1. Bias, mean squared error (MSE), and efficiency of the estimators for $\lambda$ and $\beta$ when $\lambda=0.5$ for all sampling schemes

\begin{tabular}{|c|c|c|c|c|c|c|c|c|c|c|}
\hline \multirow[b]{2}{*}{ Est. } & \multirow[b]{2}{*}{$m$} & \multirow[b]{2}{*}{$\beta$} & \multicolumn{2}{|c|}{ SRS } & \multicolumn{2}{|c|}{ RSS } & \multicolumn{2}{|c|}{ DRSS } & \multicolumn{2}{|c|}{ Efficiency } \\
\hline & & & Bias & MSE & Bias & MSE & Bias & MSE & SRS/RSS & RSS/DRSS \\
\hline \multirow[t]{21}{*}{$\hat{\lambda}$} & 6 & 0.5 & 0.1674 & 0.0333 & 0.0683 & 0.0152 & -0.0028 & 0.0085 & 2.1946 & 1.7838 \\
\hline & & 1.5 & 0.2176 & 0.0499 & 0.0888 & 0.0179 & -0.0036 & 0.0099 & 2.7885 & 1.8081 \\
\hline & & 3.0 & 0.2678 & 0.0599 & 0.1094 & 0.0315 & -0.0045 & 0.0159 & 1.9015 & 1.9811 \\
\hline & 9 & 0.5 & 0.0924 & 0.0208 & 0.0413 & 0.0124 & -0.0947 & 0.0059 & 1.6727 & 2.1019 \\
\hline & & 1.5 & 0.1664 & 0.0374 & 0.0619 & 0.0186 & -0.1515 & 0.0095 & 2.0072 & 1.9706 \\
\hline & & 3.0 & 0.2995 & 0.0673 & 0.0929 & 0.0279 & -0.2424 & 0.0151 & 2.4087 & 1.8474 \\
\hline & 10 & 0.5 & 0.0780 & 0.0104 & 0.0317 & 0.0053 & -0.0093 & 0.0028 & 1.9609 & 1.8915 \\
\hline & & 1.5 & 0.1170 & 0.0135 & 0.0475 & 0.0079 & -0.0140 & 0.0049 & 1.6994 & 1.6213 \\
\hline & & 3.0 & 0.1637 & 0.0156 & 0.0665 & 0.0111 & -0.0196 & 0.0056 & 1.4006 & 1.9861 \\
\hline & 15 & 0.5 & 0.0486 & 0.0086 & 0.0148 & 0.0041 & -0.0087 & 0.0027 & 2.0879 & 1.5242 \\
\hline & & 1.5 & 0.1020 & 0.0137 & 0.0267 & 0.0074 & -0.0139 & 0.0043 & 1.8559 & 1.7148 \\
\hline & & 3.0 & 0.2143 & 0.0206 & 0.0481 & 0.0133 & -0.0222 & 0.0069 & 1.5466 & 1.9291 \\
\hline & 20 & 0.5 & 0.0360 & 0.0051 & 0.0105 & 0.0022 & -0.0169 & 0.0012 & 2.3604 & 1.7935 \\
\hline & & 1.5 & 0.0756 & 0.0081 & 0.0220 & 0.0045 & -0.0354 & 0.0032 & 1.7984 & 1.4124 \\
\hline & & 3.0 & 0.1151 & 0.0107 & 0.0335 & 0.0099 & -0.0539 & 0.0081 & 1.0776 & 1.2222 \\
\hline & 25 & 0.5 & 0.0283 & 0.0025 & 0.0089 & 0.0014 & -0.0081 & 0.0009 & 1.7509 & 1.6452 \\
\hline & & 1.5 & 0.0537 & 0.0035 & 0.0160 & 0.0026 & -0.0105 & 0.0015 & 1.3618 & 1.7124 \\
\hline & & 3.0 & 0.1020 & 0.0059 & 0.0288 & 0.0046 & -0.0136 & 0.0019 & 1.2862 & 2.4334 \\
\hline & 30 & 0.5 & 0.0215 & 0.0023 & 0.0040 & 0.0010 & -0.0198 & 0.0006 & 2.4001 & 1.5094 \\
\hline & & 1.5 & 0.0419 & 0.0035 & 0.0078 & 0.0019 & -0.0387 & 0.0010 & 1.8462 & 1.9153 \\
\hline & & 3.0 & 0.0945 & 0.0049 & 0.0175 & 0.0043 & -0.0872 & 0.0022 & 1.1455 & 1.9866 \\
\hline
\end{tabular}


PARAMETER ESTIMATION BASED ON DRSS WITH APPLICATIONS

Table 1 (continued).

\begin{tabular}{|c|c|c|c|c|c|c|c|c|c|c|}
\hline \multirow[b]{2}{*}{ Est. } & \multirow[b]{2}{*}{$m$} & \multirow[b]{2}{*}{$\boldsymbol{\beta}$} & \multicolumn{2}{|c|}{ SRS } & \multicolumn{2}{|c|}{ RSS } & \multicolumn{2}{|c|}{ DRSS } & \multicolumn{2}{|c|}{ Efficiency } \\
\hline & & & Bias & MSE & Bias & MSE & Bias & MSE & SRS/RSS & RSS/DRSS \\
\hline \multirow[t]{21}{*}{$\hat{\beta}$} & 6 & 0.5 & 0.1440 & 0.4171 & 0.0141 & 0.2132 & -0.0614 & 0.0896 & 1.9563 & 2.3793 \\
\hline & & 1.5 & 0.3487 & 2.7577 & 0.0857 & 2.1643 & -0.4876 & 1.1728 & 1.2742 & 1.8455 \\
\hline & & 3.0 & 0.8374 & 13.0006 & 0.1876 & 8.0245 & -0.7617 & 3.2899 & 1.6201 & 2.4392 \\
\hline & 9 & 0.5 & 0.0970 & 0.1768 & 0.0180 & 0.1099 & 0.0304 & 0.0444 & 1.6086 & 2.4783 \\
\hline & & 1.5 & 0.3258 & 1.7683 & 0.0356 & 0.8537 & 0.0932 & 0.3913 & 2.0715 & 2.1815 \\
\hline & & 3.0 & 0.4624 & 6.3481 & 0.0595 & 3.3897 & 0.1689 & 1.5131 & 1.8727 & 2.2403 \\
\hline & 10 & 0.5 & 0.0754 & 0.1602 & 0.0058 & 0.0794 & -0.0528 & 0.0374 & 2.0170 & 2.1262 \\
\hline & & 1.5 & 0.2252 & 1.4242 & 0.0264 & 0.7450 & -0.3585 & 0.6990 & 1.9115 & 1.0659 \\
\hline & & 3.0 & 0.3929 & 4.9415 & 0.0517 & 2.5802 & -0.7083 & 2.2336 & 1.9151 & 1.1552 \\
\hline & 15 & 0.5 & 0.0397 & 0.0802 & 0.0049 & 0.0338 & 0.0228 & 0.0134 & 2.3717 & 2.5280 \\
\hline & & 1.5 & 0.1292 & 0.8445 & 0.0081 & 0.3119 & 0.0604 & 0.1164 & 2.7079 & 2.6784 \\
\hline & & 3.0 & 0.3018 & 3.3874 & 0.0267 & 1.2575 & 0.1334 & 0.4772 & 2.6938 & 2.6351 \\
\hline & 20 & 0.5 & 0.0290 & 0.0665 & 0.0078 & 0.0214 & -0.0241 & 0.0037 & 3.1118 & 5.8318 \\
\hline & & 1.5 & 0.1273 & 0.6224 & 0.0214 & 0.1858 & -0.1742 & 0.1215 & 3.3503 & 1.5286 \\
\hline & & 3.0 & 0.2170 & 2.2980 & 0.0009 & 0.6732 & -0.0448 & 0.3555 & 3.4137 & 1.8935 \\
\hline & 25 & 0.5 & 0.0283 & 0.0628 & -0.0022 & 0.0140 & 0.0154 & 0.0047 & 4.4935 & 2.9681 \\
\hline & & 1.5 & 0.1549 & 0.6490 & 0.0138 & 0.1152 & 0.0464 & 0.0415 & 5.6357 & 2.7718 \\
\hline & & 3.0 & 0.2629 & 2.3790 & -0.0294 & 0.4940 & 0.1030 & 0.2664 & 4.8155 & 1.8545 \\
\hline & 30 & 0.5 & 0.0310 & 0.0430 & 0.0006 & 0.0099 & -0.0058 & 0.0064 & 4.3360 & 1.5406 \\
\hline & & 1.5 & 0.0897 & 0.4256 & 0.0013 & 0.0825 & -0.0818 & 0.0332 & 5.1585 & 2.4856 \\
\hline & & 3.0 & 0.1771 & 1.5732 & -0.0023 & 0.3222 & 0.0610 & 0.1959 & 4.8832 & 1.6444 \\
\hline
\end{tabular}




\section{SABRY ET AL}

Table 2. Bias, mean squared error (MSE), and efficiency of the estimators for $\lambda$ and $\beta$ when $\lambda=1.5$ for all sampling schemes

\begin{tabular}{|c|c|c|c|c|c|c|c|c|c|c|}
\hline \multirow[b]{2}{*}{ Est. } & \multirow[b]{2}{*}{$m$} & \multirow[b]{2}{*}{$\beta$} & \multicolumn{2}{|c|}{ SRS } & \multicolumn{2}{|c|}{ RSS } & \multicolumn{2}{|c|}{ DRSS } & \multicolumn{2}{|c|}{ Efficiency } \\
\hline & & & Bias & MSE & Bias & MSE & Bias & MSE & SRS/RSS & RSS/DRSS \\
\hline \multirow[t]{21}{*}{$\hat{\lambda}$} & 6 & 0.5 & 0.5022 & 1.0829 & 0.2050 & 0.2625 & -0.0045 & 0.1893 & 5.7193 & 1.3861 \\
\hline & & 1.5 & 0.8211 & 1.1748 & 0.3352 & 0.4291 & -0.0074 & 0.3535 & 3.3229 & 1.2138 \\
\hline & & 3.0 & 1.1049 & 1.2883 & 0.4511 & 0.5774 & -0.0099 & 0.4933 & 2.6116 & 1.1705 \\
\hline & 9 & 0.5 & 0.2773 & 0.4124 & 0.1239 & 0.1192 & -0.2841 & 0.0902 & 4.5714 & 1.3211 \\
\hline & & 1.5 & 0.4437 & 0.6598 & 0.2230 & 0.2145 & -0.5682 & 0.1804 & 3.6571 & 1.1890 \\
\hline & & 3.0 & 0.7100 & 1.0557 & 0.4013 & 0.3861 & -1.1364 & 0.3608 & 2.9257 & 1.0701 \\
\hline & 10 & 0.5 & 0.2339 & 0.1212 & 0.0950 & 0.1037 & -0.0271 & 0.0526 & 1.1687 & 1.9709 \\
\hline & & 1.5 & 0.3509 & 0.1532 & 0.1425 & 0.1105 & -0.0406 & 0.0789 & 1.3864 & 1.4005 \\
\hline & & 3.0 & 0.5263 & 0.2298 & 0.2138 & 0.1526 & -0.0609 & 0.1183 & 1.5059 & 1.2894 \\
\hline & 15 & 0.5 & 0.1458 & 0.1545 & 0.0445 & 0.0444 & -0.0266 & 0.0239 & 3.4832 & 1.8521 \\
\hline & & 1.5 & 0.2624 & 0.2781 & 0.0712 & 0.0710 & -0.0346 & 0.0311 & 3.9186 & 2.2795 \\
\hline & & 3.0 & 0.4382 & 0.3568 & 0.1139 & 0.1135 & -0.0450 & 0.0405 & 3.1424 & 2.8056 \\
\hline & 20 & 0.5 & 0.1079 & 0.1143 & 0.0314 & 0.0394 & -0.0505 & 0.0214 & 2.9033 & 1.8380 \\
\hline & & 1.5 & 0.2051 & 0.2078 & 0.0596 & 0.0727 & -0.0959 & 0.0483 & 2.8583 & 1.5046 \\
\hline & & 3.0 & 0.2699 & 0.2516 & 0.0785 & 0.0928 & -0.1262 & 0.0643 & 2.7112 & 1.4428 \\
\hline & 25 & 0.5 & 0.0848 & 0.0750 & 0.0267 & 0.0284 & -0.0124 & 0.0190 & 2.6365 & 1.4957 \\
\hline & & 1.5 & 0.1611 & 0.1424 & 0.0406 & 0.0432 & -0.0206 & 0.0315 & 3.2956 & 1.3697 \\
\hline & & 3.0 & 0.3061 & 0.2706 & 0.0617 & 0.0657 & -0.0342 & 0.0524 & 4.1195 & 1.2544 \\
\hline & 30 & 0.5 & 0.0644 & 0.2524 & 0.0119 & 0.1981 & -0.0601 & 0.1002 & 1.2741 & 1.9772 \\
\hline & & 1.5 & 0.1159 & 0.4126 & 0.0215 & 0.3365 & -0.1081 & 0.1804 & 1.2262 & 1.8657 \\
\hline & & 3.0 & 0.2087 & 1.0004 & 0.0387 & 0.8037 & -0.1946 & 0.4936 & 1.2449 & 1.6281 \\
\hline
\end{tabular}


PARAMETER ESTIMATION BASED ON DRSS WITH APPLICATIONS

Table 2 (continued).

\begin{tabular}{|c|c|c|c|c|c|c|c|c|c|c|}
\hline \multirow[b]{2}{*}{ Est. } & \multirow[b]{2}{*}{$m$} & \multirow[b]{2}{*}{$\beta$} & \multicolumn{2}{|c|}{ SRS } & \multicolumn{2}{|c|}{ RSS } & \multicolumn{2}{|c|}{ DRSS } & \multicolumn{2}{|c|}{ Efficiency } \\
\hline & & & Bias & MSE & Bias & MSE & Bias & MSE & SRS/RSS & RSS/DRSS \\
\hline \multirow[t]{21}{*}{$\hat{\beta}$} & 6 & 0.5 & -0.0018 & 0.0201 & -0.0037 & 0.0115 & 0.0064 & 0.0048 & 1.7488 & 2.3941 \\
\hline & & 1.5 & 0.0075 & 0.1909 & -0.0122 & 0.1050 & -0.0140 & 0.0416 & 1.8176 & 2.5221 \\
\hline & & 3.0 & -0.0198 & 0.7386 & -0.0099 & 0.4476 & -0.0279 & 0.1665 & 1.6501 & 2.6875 \\
\hline & 9 & 0.5 & -0.0028 & 0.0130 & -0.0009 & 0.0063 & 0.0062 & 0.0032 & 2.0755 & 1.9494 \\
\hline & & 1.5 & -0.0234 & 0.1305 & -0.0067 & 0.0515 & 0.0215 & 0.0279 & 2.5327 & 1.8457 \\
\hline & & 3.0 & -0.0094 & 0.4673 & 0.0058 & 0.2076 & 0.0475 & 0.1214 & 2.2504 & 1.7107 \\
\hline & 10 & 0.5 & -0.0006 & 0.0126 & -0.0017 & 0.0045 & 0.0070 & 0.0058 & 2.8065 & 0.7694 \\
\hline & & 1.5 & -0.0060 & 0.1142 & -0.0125 & 0.0427 & 0.0099 & 0.0200 & 2.6761 & 2.1293 \\
\hline & & 3.0 & -0.0130 & 0.4097 & -0.0030 & 0.1737 & 0.0198 & 0.0940 & 2.3586 & 1.8476 \\
\hline & 15 & 0.5 & 0.0011 & 0.0080 & -0.0008 & 0.0022 & 0.0077 & 0.0011 & 3.6714 & 1.9591 \\
\hline & & 1.5 & -0.0078 & 0.0763 & -0.0041 & 0.0203 & 0.0214 & 0.0109 & 3.7524 & 1.8664 \\
\hline & & 3.0 & -0.0111 & 0.3113 & -0.0085 & 0.0781 & 0.0419 & 0.0376 & 3.9858 & 2.0745 \\
\hline & 20 & 0.5 & -0.0023 & 0.0059 & 0.0010 & 0.0012 & 0.0083 & 0.0011 & 4.8106 & 1.1691 \\
\hline & & 1.5 & -0.0033 & 0.0464 & -0.0032 & 0.0107 & 0.0500 & 0.0096 & 4.3420 & 1.1176 \\
\hline & & 3.0 & 0.0315 & 0.2025 & -0.0031 & 0.0479 & 0.0999 & 0.0382 & 4.2280 & 1.2536 \\
\hline & 25 & 0.5 & -0.0011 & 0.0059 & -0.0012 & 0.0008 & 0.0055 & 0.0004 & 7.0495 & 2.3026 \\
\hline & & 1.5 & -0.0074 & 0.0554 & -0.0011 & 0.0081 & 0.0158 & 0.0033 & 6.8690 & 2.4647 \\
\hline & & 3.0 & 0.0110 & 0.2206 & -0.0040 & 0.0314 & 0.0351 & 0.0156 & 7.0283 & 2.0145 \\
\hline & 30 & 0.5 & -0.0014 & 0.0032 & -0.0003 & 0.0007 & 0.0009 & 0.0003 & 4.3410 & 2.7631 \\
\hline & & 1.5 & 0.0027 & 0.0329 & -0.0016 & 0.0083 & 0.0073 & 0.0048 & 3.9705 & 1.7203 \\
\hline & & 3.0 & 0.0087 & 0.1012 & -0.0021 & 0.0309 & 0.0051 & 0.0159 & 3.2707 & 1.9491 \\
\hline
\end{tabular}




\section{SABRY ET AL}

Table 3. Bias, mean squared error (MSE), and efficiency of the estimators for $\lambda$ and $\beta$ when $\lambda=3$ for all sampling schemes

\begin{tabular}{|c|c|c|c|c|c|c|c|c|c|c|}
\hline \multirow[b]{2}{*}{ Est. } & \multirow[b]{2}{*}{$m$} & \multirow[b]{2}{*}{$\boldsymbol{\beta}$} & \multicolumn{2}{|c|}{ SRS } & \multicolumn{2}{|c|}{ RSS } & \multicolumn{2}{|c|}{ DRSS } & \multicolumn{2}{|c|}{ Efficiency } \\
\hline & & & Bias & MSE & Bias & MSE & Bias & MSE & SRS/RSS & RSS/DRSS \\
\hline \multirow[t]{21}{*}{$\hat{\lambda}$} & 6 & 0.5 & 1.0044 & 2.7136 & 0.4101 & 0.9264 & -0.0070 & 0.3904 & 2.9291 & 2.3728 \\
\hline & & 1.5 & 2.0015 & 3.2569 & 0.8201 & 1.8528 & -0.0141 & 0.9586 & 1.7578 & 1.9329 \\
\hline & & 3.0 & 3.0142 & 4.1867 & 1.6402 & 2.8540 & -0.0282 & 1.5459 & 1.4670 & 1.8462 \\
\hline & 9 & 0.5 & 0.5547 & 1.1495 & 0.2477 & 0.5767 & -0.5682 & 0.2608 & 1.9932 & 2.2110 \\
\hline & & 1.5 & 0.8320 & 1.7242 & 0.3964 & 0.9227 & -0.6820 & 0.4695 & 1.8686 & 1.9654 \\
\hline & & 3.0 & 1.2480 & 2.5863 & 0.6342 & 1.4763 & 0.3180 & 0.8451 & 1.7518 & 1.7470 \\
\hline & 10 & 0.5 & 0.4678 & 1.2462 & 0.1900 & 0.4847 & -0.0537 & 0.2560 & 2.5712 & 1.8930 \\
\hline & & 1.5 & 0.7017 & 1.8693 & 0.3420 & 1.8564 & -0.0698 & 1.1260 & 1.0069 & 1.6487 \\
\hline & & 3.0 & 1.0526 & 2.8039 & 0.6156 & 2.8564 & -0.0908 & 1.9586 & 0.9816 & 1.4584 \\
\hline & 15 & 0.5 & 0.2916 & 1.6180 & 0.0890 & 1.1380 & -0.0095 & 0.6579 & 1.4218 & 1.7298 \\
\hline & & 1.5 & 0.5248 & 2.9123 & 0.1335 & 2.1053 & -0.0200 & 1.3816 & 1.3833 & 1.5238 \\
\hline & & 3.0 & 0.9447 & 5.2422 & 0.2003 & 3.8948 & -0.0420 & 2.9013 & 1.3460 & 1.3424 \\
\hline & 20 & 0.5 & 0.2159 & 1.4572 & 0.0628 & 1.0775 & -0.1009 & 0.5706 & 1.3524 & 1.8883 \\
\hline & & 1.5 & 0.4318 & 2.9145 & 0.1381 & 2.3704 & -0.2522 & 1.4265 & 1.2295 & 1.6617 \\
\hline & & 3.0 & 0.8635 & 5.8289 & 0.3039 & 5.2150 & -0.6305 & 3.5663 & 1.1177 & 1.4623 \\
\hline & 25 & 0.5 & 0.1696 & 1.5998 & 0.0534 & 1.0251 & -0.0448 & 0.5012 & 1.5606 & 2.0452 \\
\hline & & 1.5 & 0.3561 & 3.3596 & 0.1334 & 2.5628 & -0.1345 & 1.5037 & 1.3109 & 1.7043 \\
\hline & & 3.0 & 0.7479 & 7.0552 & 0.3335 & 6.4071 & -0.4035 & 4.2365 & 1.1011 & 1.5124 \\
\hline & 30 & 0.5 & 0.1288 & 1.2417 & 0.0239 & 0.9352 & -0.1145 & 0.4169 & 1.3277 & 2.2432 \\
\hline & & 1.5 & 0.2963 & 2.8559 & 0.0621 & 2.4316 & -0.3322 & 1.2091 & 1.1745 & 2.0111 \\
\hline & & 3.0 & 0.6814 & 6.5686 & 0.1613 & 6.3223 & -0.9633 & 3.5064 & 1.0390 & 1.8031 \\
\hline
\end{tabular}


PARAMETER ESTIMATION BASED ON DRSS WITH APPLICATIONS

Table 3 (continued).

\begin{tabular}{|c|c|c|c|c|c|c|c|c|c|c|}
\hline \multirow[b]{2}{*}{ Est. } & \multirow[b]{2}{*}{$m$} & \multirow[b]{2}{*}{$\boldsymbol{\beta}$} & \multicolumn{2}{|c|}{ SRS } & \multicolumn{2}{|c|}{ RSS } & \multicolumn{2}{|c|}{ DRSS } & \multicolumn{2}{|c|}{ Efficiency } \\
\hline & & & Bias & MSE & Bias & MSE & Bias & MSE & SRS/RSS & RSS/DRSS \\
\hline \multirow[t]{21}{*}{$\hat{\beta}$} & 6 & 0.5 & -0.0080 & 0.0049 & -0.0013 & 0.0027 & 0.0010 & 0.0011 & 1.8407 & 2.4219 \\
\hline & & 1.5 & -0.0185 & 0.0439 & -0.0121 & 0.0271 & 0.0124 & 0.0199 & 1.6221 & 1.3615 \\
\hline & & 3.0 & -0.0400 & 0.1792 & -0.0259 & 0.1006 & -0.0778 & 0.0669 & 1.7814 & 1.5030 \\
\hline & 9 & 0.5 & -0.0013 & 0.0033 & -0.0020 & 0.0013 & 0.0040 & 0.0005 & 2.4310 & 2.4587 \\
\hline & & 1.5 & -0.0095 & 0.0299 & -0.0028 & 0.0114 & 0.0115 & 0.0048 & 2.6225 & 2.3512 \\
\hline & & 3.0 & -0.0462 & 0.1265 & -0.0161 & 0.0443 & 0.0222 & 0.0201 & 2.8547 & 2.2070 \\
\hline & 10 & 0.5 & -0.0021 & 0.0030 & -0.0012 & 0.0011 & 0.0002 & 0.0002 & 2.7812 & 4.4295 \\
\hline & & 1.5 & -0.0076 & 0.0263 & -0.0048 & 0.0101 & 0.0248 & 0.0034 & 2.6010 & 3.0004 \\
\hline & & 3.0 & -0.0182 & 0.1048 & -0.0157 & 0.0417 & -0.0925 & 0.0205 & 2.5125 & 2.0317 \\
\hline & 15 & 0.5 & -0.0028 & 0.0012 & -0.0003 & 0.0005 & 0.0032 & 0.0002 & 2.4414 & 2.6274 \\
\hline & & 1.5 & -0.0116 & 0.0122 & -0.0005 & 0.0045 & 0.0100 & 0.0016 & 2.7258 & 2.7871 \\
\hline & & 3.0 & -0.0208 & 0.0237 & -0.0086 & 0.0187 & 0.0202 & 0.0163 & 1.2638 & 1.1483 \\
\hline & 20 & 0.5 & -0.0020 & 0.0100 & -0.0005 & 0.0039 & 0.0001 & 0.0023 & 2.5811 & 1.7051 \\
\hline & & 1.5 & -0.0059 & 0.0102 & -0.0045 & 0.0030 & 0.0047 & 0.0017 & 3.3912 & 1.7322 \\
\hline & & 3.0 & -0.0177 & 0.0450 & -0.0018 & 0.0153 & -0.0573 & 0.0083 & 2.9399 & 1.8390 \\
\hline & 25 & 0.5 & -0.0010 & 0.0100 & -0.0007 & 0.0088 & 0.0030 & 0.0066 & 1.1305 & 1.3405 \\
\hline & & 1.5 & -0.0042 & 0.0022 & -0.0023 & 0.0017 & 0.0071 & 0.0005 & 1.2612 & 3.3802 \\
\hline & & 3.0 & -0.0061 & 0.0486 & -0.0015 & 0.0071 & 0.0155 & 0.0023 & 6.8451 & 3.1183 \\
\hline & 30 & 0.5 & -0.0009 & 0.0010 & 0.0002 & 0.0001 & 0.0001 & 0.0001 & 6.8424 & 1.3014 \\
\hline & & 1.5 & -0.0084 & 0.0088 & -0.0005 & 0.0016 & 0.0059 & 0.0008 & 5.6281 & 2.0894 \\
\hline & & 3.0 & -0.0099 & 0.0374 & -0.0007 & 0.0049 & -0.0326 & 0.0027 & 7.5968 & 1.8095 \\
\hline
\end{tabular}

